DOI: $10.35598 /$ mcfpga.2019.008

\title{
Review of Seventh Series FPGA Xilinx
}

\author{
Iryna Svyd \\ Department of Microprocessor Technologies and Systems \\ Kharkiv National University of Radio Electronics \\ Kharkiv, Ukraine \\ iryna.svyd@nure.ua \\ Liliia Saikivska \\ Department of Microprocessor Technologies and Systems \\ Kharkiv National University of Radio Electronics \\ Kharkiv, Ukraine \\ liliia.saikivska@nure.ua
}

\author{
Oleksandr Maltsev \\ Department of Microprocessor Technologies and Systems \\ Kharkiv National University of Radio Electronics \\ Kharkiv, Ukraine \\ aleksandr.maltsev@nure.ua \\ Oleg Zubkov \\ Department of Microprocessor Technologies and Systems \\ Kharkiv National University of Radio Electronics \\ Kharkiv, Ukraine \\ oleh.zubkov@nure.ua
}

\begin{abstract}
The work of the Xilinx FPGA of the 7th series was reviewed, as well as a comparative description of its families.
\end{abstract}

Keywords-FPGA, DSP, XADC, Xilinx, Spartan-7, Artix-7, Kintex-7, Virtex-7.

\section{INTRODUCTION}

Xilinx FPGAs are widely used today to solve problems of varying complexity. The FPGA of the 7th series of the Xilinx company combines four families [1]-[5]:

- Spartan-7,

- Artix-7,

- Kintex-7,

- Virtex-7.

These FPGA Series 7 series cover a wide range of system requirements, from low cost chips to mass production devices to ultra-high integration for high performance digital signal processing systems with high bandwidth. FPGA data has high-speed bandwidth, a large number of logical elements, the ability to process signals for a variety of digital devices [5]-[9].

\section{THE MAIN FEATURES OF THE SERIES}

The main features of the FPGA series of the 7th series of the firm Xilinx, by families [1]-[5]:

- FPGA Spartan-7 family: well-optimized for its low cost, low power consumption and high performance, $\mathrm{I} / \mathrm{O}$, has a compact placement on the board, making this type of FPGA small size;

- Artix-7 FPGA family: optimized and flexible to create low-power devices; are used to create devices that require serial-type external peripherals with high DSP bandwidth. This type of FPGA provides the lowest cost of manufacturing (total cost of materials for manufacturing) to create high-performance, sensitive to the cost of materials of digital devices;
- Kintex-7 FPGA family: Designed and optimized for the best performance of the developed digital devices, with a 2-fold improvement in performance compared to previous generations at a low price;

- Virtex-7 FPGA family: Designed and optimized for the best performance of developed digital devices, with a 2-fold improvement in performance compared to previous generations at a low price.

Comparative FPGA Performance of the 7th Series of Xilinx by Family:

- the number of a logical elements: Spartan-7 - 102000; Artix-7 - 215000; Kintex-7 - 478000; Virtex-7 - 1955000;

- the size of the RAM unit: Spartan-7 - 4.2 MB; Artix-7 - 13 MB; Kintex-7 -34 MB; Virtex-7 -68 MB;

- 3) the number of DSP Slices: Spartan-7 - 160; Artix7 - 740; Kintex-7 - 1,920; Virtex-7 - 3600;

- DSP performance. Measured in GMAC per unit time, where GMAC - Giga multiply - accumulate operations, the number of operations per second: Spartan-7 - 176 GMAC / s; Artix-7 - 929 GMAC / s; Kintex-7 - 2845 GMAC / s; Virtex-7 - 5335 $\mathrm{GMAC} / \mathrm{s}$;

- MicroBlaze processor. Measured in DMIPs, where Dhrystone Million Instructions Per second, or there are millions of operations per time unit: Spartan-7 260 DMIPs; Artix-7 - 303 DMIPs; Kintex-7 - 438 DMIPs; Virtex-7 - 441DMIPs;

- number of transceivers (devices for transmission and reception of signals): Spartan-7 - absent; Artix-7 - 16 pcs .; Kintex-7 - 32 pcs .; Virtex-7 -96 pcs .;

- transceiver speed: Spartan-7 is absent; Artix-7 - 6.6 $\mathrm{Gb} / \mathrm{s}$; Kintex-7 - 12.5 GB / s; Virtex-7 - 28.05 Gb / s; 
- Serial bandwidth: Spartan-7 is absent; Artix-7 $221 \mathrm{~Gb} / \mathrm{s}$; Kintex-7 - $800 \mathrm{~Gb} / \mathrm{s}$; Virtex-7 -2 784 GB / s;

- programmable logic controller (PLC) interface: Spartan-7 is absent; Artix-7 - x4 Gen2; Kintex-7 - x8 Gen2; Virtex-7 - x8 Gen3;

- memory interface: Spartan-7 - $800 \mathrm{Mb} / \mathrm{s}$; Artix-7 1,066 Mb / s; Kintex-7 - 1,866 Mbps; Virtex-7 -1 866 $\mathrm{Mb} / \mathrm{s}$;

- number of input / output elements: Spartan-7 - 400 units; Artix-7 - 500pcs .; Kintex-7 -500 pcs .; Virtex$7-1200$;

- the voltage consumption of input / output elements: Spartan-7 - 1.2-3.3 V; Artix-7 - 1.2-3.3V; Kintex-7 1.2-3.3V; Virtex-7 - 1.2-3.3 V.

The Xilinx Series 7 FPGA family is based on the high-k metal gate (HKMG), a modern, high-performance, lowpower 28nm technology.

The Xilinx Series 7 FPGA family provides a significant increase in system performance with up to $2.9 \mathrm{~TB} / \mathrm{s}$ throughput, about two million logical elements, as well as 5.3 TMAC / s DSPs, while consuming 50\% less power than digital devices built on the basis of the previous generation of the FPGA family, which offers a real alternative to the use of ASSP and ASIC. Xilinx's 7th Series FPGA family has: Dual 12-Bit Analog / Digital Converters (XADC) for general purpose, with a productivity of 1 million samples per second; built-in sensors for temperature and voltage control; DSP blocks with a $25 \times 18$ multiplier, 48 -bit battery and a promisor for high-performance filtering, including the possibility of optimally constructing filters with symmetric coefficients; control and synchronization units for synchronization signals, which provide high signal accuracy and low jitter level; A wide set of configuration modes, including the ability to encrypt the configuration sequence using the AES (256 bit) algorithm with HMAC / SHA-256 authentication; built-in module for detecting and correcting a one-time error. All types of cases of the 7th series are available in "leadfree" performance ( $\mathrm{Pb}$-free), and some types are also available in "lead" performance.

\section{CONCLUSIONS}

All Xilinx Series 7 FPGA chips have a common architecture that is organized in the form of columns of function blocks of the same type. Such an architecture is called an optimized silicon modular block. In addition, the Xilinx stack silicon interconnect technology used on some Virtex-7 chipsets allows the FPGA architecture to be introduced at the next structural level, making it possible to create a very high density and performance FPGA.

According to the representatives of the Xilinx company in FPGA, the 7th series embodies all the world's achievements in the field of FPGA architecture development.

\section{REFERENCES}

[1] "Spartan-7 FPGAs Data Sheet:DC and AC Switching Characteristics. Product Specification. DS189 (v1.9) March 13, 2019", Xilinx.com, 2019. [Online]. Available: https://www.xilinx.com/support/documentation/data_sheets/ds189spartan-7-data-sheet.pdf. [Accessed: 15- Jun- 2019].

[2] "Artix-7 FPGAs Data Sheet:DC and AC Switching Characteristics. Product Specification. DS181 (v1.25) June 18, 2018", Xilinx.com, 2018. [Online]. Available: https://www.xilinx.com/support/documentation/data_sheets/ds181_Ar tix_7_Data_Sheet.pdf. [Accessed: 15-Jun- 2019].

[3] "Kintex-7 FPGAs Data Sheet:DC and AC Switching Characteristics. Product Specification. DS182 (v2.18) June 28, 2019", Xilinx.com, 2019. [Online]. Available: https://www.xilinx.com/support/documentation/data_sheets/ds182_Ki ntex_7_Data_Sheet.pdf. [Accessed: 15-Jun- 2019].

[4] "Virtex-7 T and XT FPGAs Data Sheet:DC and AC Switching Characteristics. Product Specification. DS183 (v1.28) March 13, 2019", Xilinx.com, 2019. [Online]. Available: https://www.xilinx.com/support/documentation/data_sheets/ds183_Vi rtex_7_Data_Sheet.pdf. [Accessed: 15-Jun- 2019].

[5] "7 Series FPGAs Data Sheet: Overview. Product Specification. DS180 (v2.6) February 27, 2018", Xilinx.com, 2018. [Online]. Available:

https://www.xilinx.com/support/documentation/data_sheets/ds180_7S eries_Overview.pdf. [Accessed: 15- Jun- 2019].

[6] "7 Series Product Tables and Product Selection Guide", Xilinx.com, $2019 . \quad$ [Online]. Available: https://www.xilinx.com/support/documentation/selection-guides/7series-product-selection-guide.pdf. [Accessed: 15- Jun- 2019].

[7] "7 Series DSP48E1 Slice. User Guide. UG479 (v1.10) March 27, 2018", Xilinx.com, 2018. [Online]. Available: https://www.xilinx.com/support/documentation/user_guides/ug479_7 Series_DSP48E1.pdf. [Accessed: 15- Jun- 2019].

[8] "7 Series FPGAsConfiguration. User Guide. UG470 (v1.13.1) August 20, 2018", Xilinx.com, 2018. [Online]. Available: https://www.xilinx.com/support/documentation/user_guides/ug470_7 Series_Config.pdf. [Accessed: 15-Jun- 2019].

[9] V. Soloviev, Architecture of the FILM of the firm XILINX: CPLD and FPGA of the 7th series. Moscow: Hotline - Telecom, 2016, p. 392.

[10] Svyd I.V., Litvinenko O.V., Bilotserkivets O.G. Features of designing digital devices based on Xilinx FPGA in CAD Vivado HLx Design Suite. // Specialized Exhibition «KharkivProm Days. Production and efficiency". Collection of materials of the forum section "Automation, electronics and robotics. Development Strategies and Innovative Technologies ». - Kharkiv, KNURE, Exhibition Company ADT, 2019. - P. 43-44.

[11] Semenets V.V. Technology of interconnection of electronic equipment: a university textbook / V.V. Semenets, John Kratz, I.Sh. Nevlyudov, V.A. Palagin. - X.: ed. SMIT, 2005 - 432 p.

[12] V. Semenets, "Technical aspects for development laboratory base for learning FPGA and microcontroller systems," 2009 10th International Conference - The Experience of Designing and Application of CAD Systems in Microelectronics, Lviv-Polyana, 2009, pp. 145-145.

[13] S. Sakalo, V. Semenets and O. Azarhov, High frequencies in medicine (therapy and diagnostics): Teaching manual. Kharkov: KNURE; Collegium, 2005, p. 264.

[14] O. Avrunin, S. Sakalo and V. Semenetc, "Development of up-to-date laboratory base for microprocessor systems investigation," 2009 19th International Crimean Conference Microwave \& Telecommunication Technology, Sevastopol, 2009, pp. 301-302.

[15] V. Semenets, V. Kauk, O. Avrunin. "The advanced technology of remote training at the initial process" ["Vprovadjennya tehnologiy dystantsiynogo navchannya u navchalnii protses"], High School, 2009. - No. 5 .- P. 40- 45. 